\title{
COHOMOLOGY GROUPS OF LOCALLY $q$-COMPLETE MORPHISMS WITH $r$-COMPLETE BASE
}

\author{
VIOREL VÂJÂITU
}

To the memory of my Professor Martin Jurchescu

\section{Introduction.}

Vanishing theorems are important in complex analysis. One general way to obtain them was given by Andreotti and Grauert ([1]), where they showed that every $q$-complete complex space is cohomologically $q$-complete. (For the definitions, see below.)

Our main concern in this paper is to prove a variant of this theorem for families of $q$-complete spaces. We consider the following situation:

(\$) Let $\pi: X \rightarrow S$ be a holomorphic map of complex spaces such that its $f$ bres $\pi^{-1}(s), s \in S$, are q-complete. What can be said about the vanishing of the cohomology groups $H^{i}(X, \mathscr{F}), \mathscr{F} \in \operatorname{Coh}(X)$, for $i$ in a suitable range?

Simple examples show that there are holomorphic maps $\pi: X \rightarrow S$ of complex manifolds such that $S$ and all the fibes of $\pi$ are Stein, and, however, $H^{n-1}(X, \mathcal{O})$ does not vanish, where $n$ is the complex dimension of $X ; \mathrm{e}, \mathrm{g}$. $X=\mathrm{C}^{n} \backslash\{0\}, S=\mathrm{C}^{n-1}$, and $\pi$ the projection onto the first $n-1$ coordinates. Therefore, to answer our question, we have to make additional assumptions on the dependence of the family of $q$-complete spaces on the base points. In this way we are lead to locally q-complete morphins, and, the vanishing theorem holds; viz. theorem 1 in $\S 3$, which says that $X$ is cohomologically $(q+r)$-complete provided that $\pi$ and $S$ are locally $q$-complete and $r$-complete respectively. See also corollary 2 in $\$ 3$.

As consequences (viz., theorem 2 and corollaries 3, 4, and 6), one gets vanishing theorems for the cohomology of locally $q$-complete open sets in $r$ complete spaces.

Received April 12, 1995. 
Particular cases of our results were treated by various authors; Ballico ([2]), Bolondi ([3]), and Jennane ([6], [7], [8]).

Throughout this paper all complex spaces are assumed to be reduced and with countable topology.

\section{Preliminaries.}

Let $D$ be an open subset of $C^{n}$ and $f \in C^{\infty}(D, \mathrm{R})$. Let $z_{1}, \ldots, z_{n}$ be the complex coordinates of $\mathrm{C}^{n}$. For every point $w \in D$, the quadratic form

$$
\mathrm{C}^{n} \ni \xi \mapsto L(f, w)(\xi):=\sum_{i, j=1}^{n} \frac{\partial^{2} f}{\partial z_{i} \partial \bar{z}_{j}}(w) \xi_{i} \bar{\xi}_{j} \in \mathrm{R},
$$

is called the Levi form of $f$ at $w$. We say that $f$ is $q$-convex on $D$ if its Levi form $L(f, w)$ has at least $n-q+1$ positive eigenvalues for every $w \in D$.

Let $X$ be a complex space. A function $\varphi: X \rightarrow \mathrm{R}, X$ a complex space, is said to be $q$-convex if for each point $x \in X$ there is an holomorphic imbedding $\iota: U \rightarrow \hat{U}, U \ni x, \hat{U} \subset \mathrm{C}^{n}$ open, and a $q$-convex function $\hat{\varphi} \in C^{2}(\hat{U}, \mathbf{R})$ which extends $\left.\varphi\right|_{U}$.

We say that $X$ is $q$-complete (resp. $q$-convex) if there exists a smooth exhaustion function $\varphi: X \rightarrow \mathrm{R}$ which is $q$-convex on $X \backslash K$ for some suitable compact subset $K$ of $X$ ). The normalization is such that Stein spaces correspond to 1-complete spaces.

Finitely many $q$-convex functions $\varphi_{1}, \ldots, \varphi_{k}$, on $X$ have the same positivity directions if for each point $x_{0} \in X$ there are: an holomorphic imbedding $\iota: U \rightarrow \hat{U}, U \ni x_{o}, \hat{U} \subset C^{n}$ open; $q$-convex extensions $\hat{\varphi}_{j}$ of $\varphi_{j}, j=1, \ldots, k$; and a complex vector subspace $E$ of $C^{n}$ of dimension at least $n-q+1$ such that all the Levi forms $L\left(\hat{\varphi}_{1}, z_{o}\right), \ldots, L\left(\hat{\varphi}_{k}, z_{o}\right), z_{o}=\iota\left(x_{0}\right)$ are positive definite when restricted to $E$.

The motivation for this notion is the following: Let $\varphi_{1}$ and $\varphi_{2}$ be $q$-convex with the same positivity directions. Then $\varphi_{1}+\varphi_{2}$ is again $q$-convex and $\max \left(\varphi_{1}, \varphi_{2}\right)$ can be approximated in the $C^{o}$-topology by $q$-convex functions ([16]).

Definition. Let $\pi: X \rightarrow S$ be a holomorphic map between complex spaces. (We consider $S$ as a space of parameters.)

$(\bullet)$ We say that $\pi$ is: $q$-convex (resp. $q$-complete) if there exists a smooth function $\varphi: X \rightarrow \mathbf{R}$ and a real number $c_{\sharp}$ (resp. $c_{\sharp}=-\infty$ ) such that:

(i) $\varphi$ is $q$ convex on the open set $\left\{x \in X ; \varphi(x)>c_{\sharp}\right\}$;

(ii) For every real number $\lambda$, the restriction of $\pi$ from $\{x \in X ; \varphi(x) \leq \lambda\}$ to $S$ is proper. 
We call $\varphi$ the exhaustion function of $X$ and $c_{\sharp}$ the convexity bound.

$(\bullet)$ We say that $\pi$ is: locally $q$-convex (resp. locally $q$-complete) if every point $s \in S$ has an open neighborhood $U$ such that the restriction of $\pi$ from $\pi^{-1}(U)$ to $U$ is $q$-convex (resp. $q$-complete).

REMARKS. 1) If $\pi: X \rightarrow S$ is locally $q$-convex (resp. locally $q$-complete), then its fibres $X_{s}:=\pi^{-1}(s), s \in S$, are $q$-convex (resp. $q$-complete) complex spaces. In particular, if $S$ is a point, the definitions of $q$-complete and $q$ convex spaces are regained.

2) One verifies readily that $\pi: X \rightarrow S$ is locally $q$-complete if, and only if, every point $s \in S$ has a neighborhood $U$ such that $\pi^{-1}(U)$ is $q$-complete. (An analogous situation does not occur for $q$-convex mappings as the example 1 from below shows).

Moreover, in this case, if $V \subset U$ is an arbitrary Stein open set, then $\pi^{-1}(V)$ is again $q$-complete.

3) Also, it is easy to check that if $\pi$ is $q$-complete and $S$ is $r$-complete (resp. $r$-convex), then $X$ is $(q+r-1)$-complete (resp. $(q+r-1)$-convex). (This does not hold for locally $q$-complete mappings as the example 2 in $\S 3$ shows.)

4) Locally 1-complete morphisms are also called locally Stein morphisms ([6], [7]).

EXAMPLE 1. Let $A \subset \mathrm{C}^{n}$ be a closed submanifold of pure dimension $d \leq n-2$, and $\pi: X \rightarrow \mathrm{C}^{n}$ the blowing-up of $\mathrm{C}^{n}$ at $A$. Then $\pi$ is 1-convex.

Indeed, if $A=\left\{f_{1}=\ldots=f_{m}=0\right\}$ for some holomorphic functions $f_{1}, \ldots, f_{m}$ on $\mathrm{C}^{n}$, we set $h: \mathrm{C}^{n} \rightarrow \mathrm{R}$ by $h(z)=\left(\left|f_{1}(z)\right|^{2}+\ldots+\left|f_{m}(z)\right|^{2}\right)$ $\exp \left(\|z\|^{2}\right)$. Then, $\varphi=h(\pi)$ together with $c_{\sharp}=0$ as convexity bound show the 1-convexity of $\pi$. However, if $d>0$, then for each open subset $U \subset \mathrm{C}$ with $U \cap A \neq \emptyset, \pi^{-1}(U)$ is not 1-convex. (In fact, not even $(n-d-1)$-convex.)

By extending the usual notion of Runge domains in Stein spaces, we say that an open subset $D$ of a complex space $X$ is $q$-Runge if for every compact set $K \subset D$ there is a $q$-convex exhaustion function $\varphi: X \rightarrow \mathrm{R}$ (which may depend on $K$ ) such that

$$
K \subset\{x \in X ; \varphi(x)<0\} \Subset D .
$$

(Note that $X$ is $q$-complete if and only if the empty set is $q$-Runge in $X$.) With this definition, we reinterprete a result due to Andreotti and Grauert ([1]).

Proposition 1. Let $D$ be a $q$-Runge domain in a q-complete complex space 
$X$. Then, for every coherent analytic sheaf $\mathscr{F}$ on $X, H^{i}(D, \mathscr{F})$ vanishes for $i \geq q$, and the restriction map $H^{q-1}(X, \mathscr{F}) \rightarrow H^{q-1}(D, \mathscr{F})$ has dense range for the natural topology.

A complex space $X$ is said to be cohomologically $q$-complete (resp. cohomologically q-convex) if the cohomology groups $H^{i}(X, \mathscr{F}), \mathscr{F} \in \operatorname{Coh}(X)$ vanish (resp. are finite dimensional complex vector spaces) for every $i \geq q$.

An open subset $D$ of a complex space $X$ is said to be locally q-complete if for each point $x \in \partial D$ there exists an open neighborhood $U$ of $X$ such that $U \cap D$ is $q$-complete. Equivalently, the inclusion map $j: D \rightarrow X$ is locally $q$ complete.

In the sequel, topological vector spaces are such that its zero element has a countable base of open neighborhoods. For such a topological vector space $E$ we denote by $E_{\text {sep }}$ the separated topological space associated with $E$, namely; the quotient of $E$ modulo the closure of its zero-element. The following result is evident.

LEMMA 1. Let $u: E \rightarrow F$ be a continuous map of topological vector spaces. The following statements are equivalent one another:

a) $u$ has the lifting property of sequences, i.e., for every sequence $\left\{f_{n}\right\} \subset u(E)$ with $f_{n} \rightarrow 0$ there is another sequence $\left\{e_{n}\right\} \subset u(E), e_{n} \rightarrow 0$ and $u\left(e_{n}\right)=f_{n}, n \geq 1$.

b) $u$ is quasi-open, i.e., the induced map $u^{\prime}: E \rightarrow u(E)$ is open where $u(E) \subset F$ is endowed with the trace topology which comes from $F$.

By diagram chasing, the following is a consequence of the preceding lemma.

Corollary 1. Assume we have a commutative diagram of topological vector spaces with exact rows

$$
\begin{array}{ccccc}
E & \rightarrow & F & \rightarrow & G \\
a \downarrow & & b \downarrow & & c \downarrow \\
E^{\prime} & \rightarrow & F^{\prime} & \stackrel{v}{\rightarrow} & G^{\prime}
\end{array}
$$

where $a, b, c, v$ are continuous linear maps. Suppose $a$ and c have dense range. Then $b$ has dense range provided that $v$ is quasi-open.

Let $X$ be a complex space. A Stein open covering $\mathscr{U}=\left(U_{i}\right)_{i \in I}$ of $X$ is said to be a special covering of $X$ if $\mathscr{U}$ is a countable base of open subsets of $X$. If $D \subset X$ is open, we let $\mathscr{U}_{\mid D}:=\{U \in \mathscr{U} ; U \subseteq D\}$. Obviously, $\mathscr{U}_{\mid D}$ is a special covering of $D$.

Now, we let $\mathscr{F} \in \operatorname{Coh}(X)$. Since the spaces of Čech cochains $C^{p+1}(\mathscr{U}, \mathscr{F})$, $p=0,1, \ldots$, are Fréchet spaces, and the coboundary maps 
$\delta=\delta^{p}: C^{p}(\mathscr{U}, \mathscr{F}) \rightarrow C^{p+1}(\mathscr{U}, \mathscr{F})$ are continuous, the spaces of cocycles $Z^{p}(\mathscr{U}, \mathscr{F}):=\operatorname{Ker} \delta^{p}$ are also Fréchet spaces.

Thus we have a simple:

LEMMA 2. For every non-negative integer $p$, the following statements are equivalent:

a) The restriction map $H^{p}(X, \mathscr{F}) \rightarrow H^{p}(D, \mathscr{F})$ has dense range.

b) The restriction map $Z^{p}(\mathscr{U}, \mathscr{F}) \rightarrow Z^{p}\left(\mathscr{U}_{\mid D}, \mathscr{F}\right)$ has dense range for any special covering $\mathscr{U}$ of $X$.

c) Statement b) holds for one special covering $\mathscr{V}$ of $X$.

The next lemma is probably well known, but since we did not found any reference in the literature we give it here.

Lemma 3. Let $X$ be a complex space and $X_{1}, X_{2}$ be open subsets. Let also $\mathscr{F} \in \operatorname{Coh}(X)$ and $p$ a non-negative integer such that $H^{p}\left(X_{1} \cap X_{2}, \mathscr{F}\right)$ is Hausdorff. Then the natural map which comes from the Mayer-Vietoris sequence

$$
H^{p}\left(X_{1} \cup X_{2}, \mathscr{F}\right) \rightarrow H^{p}\left(X_{1}, \mathscr{F}\right) \oplus H^{p}\left(X_{2}, \mathscr{F}\right)
$$

is quasi-open. In particular, this holds if $H^{p}\left(X_{1} \cap X_{2}, \mathscr{F}\right)$ vanishes.

Proof. Since the case $p=0$ is clear, we may assume $p \geq 1$; and without any loss of generality, let $X=X_{1} \cup X_{2}$. We break the proof into three steps.

Step 1. There is an special covering $\mathscr{U}=\left(U_{i}\right)_{i \in I}$ of $X$ such that for the next three sets of indices $I_{1}:=\left\{i \in I ; U_{i} \subset X_{1}\right\}, I_{2}:=\left\{i \in I ; U_{i} \subset X_{2}\right\}$ and $I_{12}:=\left\{i \in I ; U_{i} \subset X_{1} \cap X_{2}\right\}$, it holds: (\$) If $i \in I_{1} \backslash I_{12}$ and $j \in I_{2} \backslash I_{12}$, then $\overline{U_{i}} \cap \overline{U_{j}}=\emptyset$.

Indeed, first select $\mathscr{U}_{12}$ an arbitrary special covering of $X_{1} \cap X_{2}$. Then there are disjoint open sets $D_{1} \subset X_{1}$ and $D_{2} \subset X_{2}$ such that $X_{1} \backslash X_{2} \subset D_{1}$ and $X_{2} \backslash X_{1} \subset D_{2}$. Further choose $\mathscr{U}_{1}$ and $\mathscr{U}_{2}$ special coverings of $D_{1}$ and $D_{2}$ respectively. Finally, set $\mathscr{U}=$ the collection of all open sets from $\mathscr{U}_{1}, \mathscr{U}_{2}$ and $\mathscr{U}_{12}$. Note that, if $U_{i_{1}} \cap \ldots \cap U_{i_{s}} \neq \emptyset$, then $U_{i_{1}} \cup \ldots \cup U_{i_{s}}$ is either contained in $X_{1}$ or in $X_{2}$.

Step 2. With the notations from above, there is a commutative diagram

$$
\begin{array}{ccccc}
C^{p-1}\left(\mathscr{U}_{12}, \mathscr{F}\right) \oplus Z^{p}(\mathscr{U}, \mathscr{F}) & \stackrel{u}{\rightarrow} & Z^{p}\left(\mathscr{U}_{1}, \mathscr{F}\right) \oplus Z^{p}\left(\mathscr{U}_{2}, \mathscr{F}\right) & \stackrel{v}{\rightarrow} & Z^{p}\left(\mathscr{U}_{12}, \mathscr{F}\right) \\
\alpha \downarrow & & \beta \downarrow & & \gamma \downarrow \\
H^{p}\left(X_{1} \cup X_{2}, \mathscr{F}\right) & \stackrel{u^{\prime}}{\rightarrow} & H^{p}\left(X_{1}, \mathscr{F}\right) \oplus H^{p}\left(X_{2}, \mathscr{F}\right) & \stackrel{v^{\prime}}{\rightarrow} & H^{p}\left(X_{1} \cap X_{2}, \mathscr{F}\right),
\end{array}
$$

where $\beta, \gamma, u^{\prime}, v^{\prime}$ are the natural maps, $\alpha$ is obtained by extending with zero the natural map $Z^{p}(\mathscr{U}, \mathscr{F}) \rightarrow H^{p}\left(X_{1} \cup X_{2}, \mathscr{F}\right)$, and $v, u$ are to be constructed as follows: 
For

$$
\left(\xi_{1}, \xi_{2}\right) \in Z^{p}\left(\mathscr{U}_{1}, \mathscr{F}\right) \oplus Z^{p}\left(\mathscr{U}_{2}, \mathscr{F}\right)
$$

set

$$
v\left(\xi_{1}, \xi_{2}\right):=\xi_{2_{\mid X_{1} \cap X_{2}}}-\xi_{1_{\mid X_{1} \cap X_{2}}}
$$

For

$$
(\eta, \xi) \in C^{p-1}\left(\mathscr{U}_{12}, \mathscr{F}\right) \oplus Z^{p}(\mathscr{U}, \mathscr{F})
$$

set

$$
u(\eta, \xi):=\left(\xi_{\mid X_{1}}, \xi_{\mid X_{2}}+\delta \tilde{\eta}\right)
$$

where $\tilde{\eta} \in C^{p-1}\left(\mathscr{U}_{12}, \mathscr{F}\right)$ is the trivial extension of $\eta$ to $C^{p-1}\left(\mathscr{U}_{2}, \mathscr{F}\right)$.

We claim that: $\operatorname{Im} u=v^{-1}(\operatorname{Ker} \gamma)$.

Only " $\supseteq$ " needs a proof. Let $\xi_{1} \in Z^{p}\left(\mathscr{U}_{1}, \mathscr{F}\right), \xi_{2} \in Z^{p}\left(\mathscr{U}_{2}, \mathscr{F}\right)$ and $\eta \in C^{p-1}\left(\mathscr{U}_{12}, \mathscr{F}\right)$ be such that $\xi_{2_{\mid X_{1} \cap x_{2}}}-\xi_{1_{\mid X_{1} \cap x_{2}}}=\delta \eta$. Now let $\tilde{\eta} \epsilon$ $C^{p-1}\left(\mathscr{U}_{2}, \mathscr{F}\right)$ be the trivial extension of $\eta$. Then $\left(\xi_{2}-\delta \tilde{\eta}\right)_{\mid X_{1} \cap X_{2}}=\xi_{1_{\mid X_{1} \cap X_{2}}}$. We define $\xi \in Z^{p}(\mathscr{U}, \mathscr{F})$ by

$$
\left\{\begin{array}{l}
\xi_{\mid X_{1}}:=\xi_{1} \\
\xi_{\mid X_{2}}:=\xi_{2}-\delta \tilde{\eta} .
\end{array}\right.
$$

This is well-defined because of (\$), and we have $u(\eta, \xi)=\left(\xi_{1}, \xi_{2}\right)$.

Step 3. Here we conclude the proof of the lemma. By hypothesis Ker $\gamma$ is separated; and by the above claim $u$ has closed image since $v$ is continuous. On the one hand, by the Banach open theorem $u$ is quasi open. On the other hand, from the commutativity of the left square in the diagram from step 2 $u^{\prime}$ results quasi-open.

For the bumping techniques we shall need the next

LeMma 4. Let $Y$ be a complex space and $Y_{1}, Y_{2}$ open subsets such that $Y=Y_{1} \cup Y_{2}$. Let $\mathscr{F} \in \operatorname{Coh}(Y)$ and $q$ a positive integer. Then $H^{q}(Y, \mathscr{F})_{\text {sep }}$ vanishes if the subsequent two statements hold.

(a) $H^{q}\left(Y_{1}, \mathscr{F}\right)_{\text {sep }}$ vanishes.

(b) $Y_{1} \cap Y_{2}$ is q-Runge in $Y_{2}$.

PROOF. We let $\mathscr{U}$ be a special covering of $Y$ and set: $\mathscr{U}_{1}:=\mathscr{U}_{Y_{1}}, \mathscr{U}_{2}:=$ $\left.\mathscr{U}\right|_{Y_{2}}$, and $\mathscr{U}_{12}:=\left.\mathscr{U}\right|_{Y_{1} \cap Y_{2}}$. Statement (a) means that the natural coboundary map $\delta: C^{q-1}\left(\left.\mathscr{U}\right|_{Y_{1}}, \mathscr{F}\right) \rightarrow Z^{q}\left(\left.\mathscr{U}\right|_{Y_{1}}, \mathscr{F}\right)$ has dense range. We have to show that $\delta: C^{q-1}(\mathscr{U}, \mathscr{F}) \rightarrow Z^{q}(\mathscr{U}, \mathscr{F})$ has dense range. For this, we consider the map 


$$
\rho: C^{q-1}\left(\mathscr{U}_{1}, \mathscr{F}\right) \oplus C^{q-1}\left(\mathscr{U}_{2}, \mathscr{F}\right) \rightarrow Z^{q}\left(\mathscr{U}_{12}, \mathscr{F}\right)
$$

defined by $\rho\left(\xi_{1}, \xi_{2}\right):=\delta\left(\left.\xi_{1}\right|_{Y_{12}}-\left.\xi_{2}\right|_{Y_{12}}\right)$ where $Y_{12}:=Y_{1} \cap Y_{2}$.

We claim that $\rho$ is surjective. To show this, note that by proposition 1 one has $Z^{q}\left(\mathscr{U}_{12}, \mathscr{F}\right)=\delta C^{q-1}\left(\mathscr{U}_{12}, \mathscr{F}\right)$. Now let $\alpha \in C^{q-1}\left(\mathscr{U}_{12}, \mathscr{F}\right)$ and consider $\xi_{1}:=\tilde{\alpha}, \xi_{2}:=0$, where $\tilde{\alpha}$ is the trivial extension of $\alpha$ to $C^{q-1}\left(\mathscr{U}_{1}, \mathscr{F}\right)$. Then $\rho\left(\xi_{1}, \xi_{2}\right)=\delta \alpha$, whence the surjectivity of $\rho$; hence $\rho$ is open. Consequently, it has the lifting property of sequences. In order to finish the proof of the lemma, we fix $\xi \in Z^{q}(\mathscr{U}, \mathscr{F})$ arbitrary. Then choose a sequence $\left\{\theta_{1}^{(n)}\right\}_{n} \subset C^{q-1}\left(\mathscr{U}_{1}, \mathscr{F}\right),\left.\delta \theta_{1}^{(n)} \rightarrow \xi\right|_{Y_{1}}$. Let $\theta_{2} \in C^{q-1}\left(\mathscr{U}_{2}, \mathscr{F}\right), \delta \theta_{2}=\left.\xi\right|_{Y_{2}}$ (Note that $Y_{2}$ is $q$-complete.) Therefore in $Z^{q}\left(\mathscr{U}_{12}, \mathscr{F}\right)$ one has $\delta\left(\left.\theta_{1}^{(n)}\right|_{Y_{12}}\right)$ $\delta\left(\left.\theta_{2}\right|_{Y_{12}}\right) \rightarrow 0$

Now choose sequences $\left\{\alpha_{1}^{(n)}\right\}_{n} \subset C^{q-1}\left(\mathscr{U}_{1}, \mathscr{F}\right)$ and $\left\{\alpha_{2}^{(n)}\right\}_{n} \subset C^{q-1}\left(\mathscr{U}_{2}, \mathscr{F}\right)$ which converge to zero and such that

$$
\delta\left(\alpha_{1}^{(n)}\right)-\delta\left(\alpha_{2}^{(n)}\right)=\delta\left(\theta_{1}^{(n)}\right)-\delta\left(\theta_{2}\right) .
$$

Thus $\quad u^{(n)}:=\alpha_{2}^{(n)}-\theta_{2}-\alpha_{1}^{(n)}+\theta_{1}^{(n)} \in Z^{q}\left(\mathscr{U}_{12}, \mathscr{F}\right) . \quad$ Now $\quad Z^{q-1}\left(\mathscr{U}_{2}, \mathscr{F}\right) \rightarrow$ $Z^{q-1}\left(\mathscr{U}_{12}, \mathscr{F}\right)$ has dense range from (b), proposition 1, and lemma 2 . Thus there exists a sequence

$$
\left\{h^{(n)}\right\}_{n} \subset Z^{q-1}\left(\mathscr{U}_{2}, \mathscr{F}\right), \text { with }\left.h^{(n)}\right|_{Y_{12}}-u^{(n)} \rightarrow 0
$$

on $Y_{12}$. Let $\tilde{h}_{1}^{(n)}, \tilde{u}^{(n)}$ be the trivial extensions to $C^{p-1}\left(\mathscr{U}_{1}, \mathscr{F}\right)$. Define a sequence $\left\{\eta^{(n)}\right\}_{n}$ in $C^{q-1}(\mathscr{U}, \mathscr{F})$ by:

$$
\eta^{(n)}:= \begin{cases}\theta_{1}^{(n)}-\alpha_{1}^{(n)}+\tilde{h}_{1}^{(n)}-\tilde{u}^{(n)} & \text { on } \mathscr{U}_{1} \\ \theta_{2}-\alpha_{2}^{(n)}+h_{2}^{(n)}, & \text { on } \mathscr{U}_{2}\end{cases}
$$

Then $\delta \eta^{(n)} \rightarrow \xi$, whence the lemma.

We conclude this paragraph with the next:

LEMMA 5 Let $\pi: X \rightarrow S$ be a holomorphic map of complex spaces and $D \Subset S$ an open subset such that $\pi^{-1}(D)$ is q-complete. Let also $\varphi_{1}, \varphi_{2} \in C^{\infty}(S, R)$ be two r-convex functions with the same positivity directions. Set $U_{i}:=\left\{s \in D ; \varphi_{i}(s)<0\right\}, i=1,2$, and $p=q+r-1$. Then $\pi^{-1}\left(U_{1} \cap U_{2}\right)$ is p-complete and p-Runge in $\pi^{-1}\left(U_{2}\right)$.

Proof. Let $\psi: \pi^{-1}(D) \rightarrow(0, \infty)$ be $q$-convex and exhaustive. For every real number $C>0$ define a family of continuous exhaustion functions $\psi_{C}: \pi^{-1}\left(U_{2}\right) \rightarrow \mathrm{R}$ by

$$
\psi_{C}:=\psi-1 /\left(\varphi_{2} \circ \pi\right)+C \cdot \max \left(\varphi_{1} \circ \pi, \varphi_{2} \circ \pi\right) .
$$


If $K \subset \pi^{-1}\left(U_{1} \cap U_{2}\right)$ is a compact set, then, with a large enough $C>0$, we get

$$
K \subset\left\{\psi_{c}<0\right\} \Subset \pi^{-1}\left(U_{2}\right) .
$$

A suitable smooth $p$-convex approximation of $\psi_{C}$ in the $C^{o}$-topology ([16]) enables us to conclude that $\pi^{-1}\left(U_{1} \cap U_{2}\right)$ is $p$-Runge in $\pi^{-1}\left(U_{2}\right)$.

The $p$-completeness of $\pi^{-1}\left(U_{1} \cap U_{2}\right)$ results if one approximates the function

$$
\psi-1 / \max \left(\varphi_{1} \circ \pi, \varphi_{2} \circ \pi\right) \in C^{o}\left(\pi^{-1}\left(U_{1} \cap U_{2}\right), \mathrm{R}\right)
$$

in the $C^{o}$-topology by smooth $p$-convex functions ([16]).

\section{The results.}

Here is our relative vanishing theorem for families of $q$-complete complex spaces.

THEOREM 1. Let $\pi: X \rightarrow S$ be a locally $q$-complete morphism of complex spaces. If $S$ is $r$-complete, then $X$ is cohomologically $(q+r)$-complete. Moreover, $H^{q+r-1}(X, \mathscr{F})_{\text {sep }}$ vanishes for every coherent analytic sheaf $\mathscr{F}$ on $X$.

PRoof. We consider ([16]) a $r$-convex exhaustion function $h: S \rightarrow \mathrm{R}$ such that for every real number $\lambda$ if $S(\lambda):=\{s \in S ; h(s)<\lambda\}$, then the set

$$
\{s \in S ; h(s)=\lambda\} \backslash \partial S(\lambda)
$$

contains at most one point. Correspondingly, define the sets $X(\lambda):=$ $\pi^{-1}(S(\lambda))$.

Put $p=q+r-1$ and let $\mathscr{F} \in \operatorname{Coh}(X)$. We claim that for every pair of real numbers $\lambda<\mu$ we have:

(a) The restriction $H^{p}(X(\mu), \mathscr{F}) \rightarrow H^{p}(X(\lambda), \mathscr{F})$ has dense range;

(b) $H^{i}(X(\lambda), \mathscr{F})$ vanishes for all $i \geq q+r$;

(c) $H^{p}(X(\mu), \mathscr{F})_{\text {sep }}$ vanishes.

First we show that (a) holds. For this, we define $T \subseteq \mathrm{R}$ to be the set of all real numbers $\mu$ such that the restriction map $H^{p}(X(\mu), \mathscr{F}) \rightarrow H^{p}(X(\lambda), \mathscr{F})$ has dense image for every real number $\lambda$ with $\lambda<\mu$.

Obviously, $T$ is not empty. In fact if $\mu_{*}:=\min \{h(s) ; s \in S\}$, then one sees easily that $\left(-\infty, \mu_{*}\right] \subset T$. Also, by lemma 2 and a standard argument of Fréchet spaces, $T$ is closed. To prove $T$ is open, we use the bumping technique of Andreotti and Grauert. To begin with, fix some $\mu \in T$. We shall find $\epsilon_{o}>0$ such that $\mu_{o}+\epsilon_{o} \in T$. Recall that $\left\{h=\mu_{o}\right\} \backslash \partial S\left(\mu_{o}\right)$ is empty or 
equals $\left\{s_{o}\right\}$ for some $s_{o} \in S$. We treat only the second case since the first one is similar (so we omit its proof).

Let $U \subset S$ be a Stein open neighborhood of $s_{o}$ such that $\pi^{-1}(U)$ is $q$ complete and $\bar{U} \cap \overline{S\left(\mu_{o}\right)}=\emptyset$. Choose finitely many Stein open sets $\left\{U_{j}\right\}, j=1, \ldots, k$, disjoint from $U$, which cover $\partial S\left(\mu_{o}\right)$ and such that $\pi^{-1}\left(U_{j}\right)$ are $q$-complete. Let $V_{j} \Subset U_{j}$ be also open Stein sets such that $\left\{V_{j}\right\}_{j}$ still covers $\partial S\left(\mu_{o}\right)$. Then select $\left\{\rho_{j}\right\} \in C^{\infty}\left(U_{j}, \mathrm{R}\right), \rho_{j} \geq 0$, and $\rho_{j} \equiv 1$ on $V_{j}, j=1, \ldots, k$. Define smooth functions $h_{j}: X \rightarrow \mathrm{R}$ by

$$
h_{j}:=h-\sum_{v=1}^{j} c_{\nu} \rho_{v}, j=1, \ldots, k,
$$

where $c_{v}>0$ are small enough constants such that $h_{o}:=h, h_{1}, \ldots, h_{k}$, are $r$ convex with the same positivity directions. Set

$$
S_{j}:=\left\{s \in S ; h_{j}(s)<\mu_{o}\right\}, j=1, \ldots, k \text { and } S_{o}:=S\left(\mu_{o}\right) .
$$

Obviously, $S_{j} \backslash S_{j-1} \Subset U_{j}$. Also since $h$ is proper, there exists $\epsilon_{o}>0$ with $S\left(\mu_{o}+\epsilon_{o}\right) \subseteq S_{k} \cup U$. We define for an arbitrary real number $\mu$ and integer $j=0, \ldots, k$, the set

$$
X_{j}(\mu):=\pi^{-1}\left(S_{j} \cap S(\mu)\right) .
$$

Since $S(\mu)=\left(S(\mu) \cap S_{k}\right) \cup(S(\mu) \cap U)$ we get: $X(\mu)=X_{k}(\mu) \cup V(\mu)$, where $V(\mu):=\pi^{-1}(S(\mu) \cap U)$ is $p$-complete by lemma 4 . Moreover, we remark that

$$
X_{k}(\mu) \cap V(\mu) \text { is } p \text {-Runge in } V(\mu) .
$$

Therefore $\quad H^{p}(X(\mu), \mathscr{F})=H^{p}\left(X_{k}(\mu), \mathscr{F}\right) \oplus H^{p}(V(\mu), \mathscr{F})=H^{p}\left(X_{k}(\mu), \mathscr{F}\right)$. Now fix $\mu$ and $\lambda$ with $\mu_{o}<\mu \leq \mu_{o}+\epsilon_{o}$ and $\lambda<\mu$. To get (a) we show inductively on $j$ that

$$
H^{p}\left(X_{j}(\mu), \mathscr{F}\right) \rightarrow H^{p}\left(X_{j}(\lambda), \mathscr{F}\right)
$$

has dense range. For $j=0$ this is clear since $\mu_{o} \in T$. Now let $j \geq 1$. We have

$$
X_{j}(\mu)=X_{j-1}(\mu) \cup V_{j}(\mu)
$$

where $V_{j}(\mu):=\pi^{-1}\left(U_{j} \cup S(\mu)\right)$. Note also that

$$
X_{j-1}(\mu) \cap V_{j}(\mu) \text { is } p \text {-complete and } p \text {-Runge in } V_{j}(\mu) \text {. }
$$

This is a consequence of lemma 5 for $D:=U_{j}, \varphi_{1}:=h_{j-1}-\mu_{o}$, and $\varphi_{2}:=h-\mu$. Now, from Mayer-Vietoris sequence, one gets the subsequent commutative diagram with exact rows (Note that $V_{j}(\mu)$ and $V_{j}(\lambda)$ are $p$ complete) 


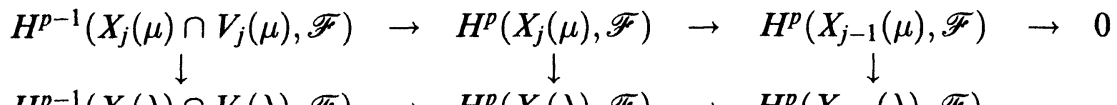

$$
\begin{aligned}
& H^{p-1}\left(X_{j}(\lambda) \cap V_{j}(\lambda), \mathscr{F}\right) \rightarrow H^{p}\left(X_{j}(\lambda), \mathscr{F}\right) \rightarrow H^{p}\left(X_{j-1}(\lambda), \mathscr{F}\right) .
\end{aligned}
$$

Lemma 3 applied for $X_{1}:=X_{j-1}(\lambda), X_{2}:=V_{j}(\lambda)$; the $p$-completeness of $X_{2}$ and $X_{1} \cap X_{2}$; and corollary 1 yield $(\nabla)$.

Statement (b) is similar to (a), (and, in fact easier) so we omit its proof.

In order to prove statement (c), one chooses a a special covering $\mathscr{U}$ of $X$ and showes that the coboundary map

$$
\delta: C^{q+r-2}\left(\mathscr{U}_{\left.\right|_{X(\mu)}}, \mathscr{F}\right) \rightarrow Z^{q+r-1}\left(\mathscr{U}_{\left.\right|_{X(\mu)}}, \mathscr{F}\right)
$$

has dense range. This folows by lemma 4 and the proof of (a) given above by using (1) to (4).

Now the cohomological statement of the theorem follows in a standard way, because for all $v=0,1, \ldots$, we have that $H^{i}(X(v), \mathscr{F})$ vanishes for all $i \geq q+r$, and the restriction maps $\left.H^{q+r-1}(X(v+1), \mathscr{F}) \rightarrow H^{q+r-1}(v), \mathscr{F}\right)$ have dense image.

In order to obtain "the moreover", note that for every special covering $\mathscr{U}$ of $X$, the restriction maps $C^{p-1}\left(\mathscr{U}_{\left.\right|_{X(v+1)}}, \mathscr{F}\right) \rightarrow C^{p-1}\left(\mathscr{U}_{\left.\right|_{X(v)}}, \mathscr{F}\right), v=0,1, \ldots$, are surjective. Also by lemma 2 and (a) from above, the restrictions $Z^{p}\left(\mathscr{U}_{\left.\right|_{X(v+1)}}, \mathscr{F}\right) \rightarrow Z^{p}\left(\mathscr{U}_{\left.\right|_{X(v)}}, \mathscr{F}\right)$ have dense range. A standard argument of Fréchet spaces yields that the coboundary map $\delta: C^{p-1}(\mathscr{U}, \mathscr{F}) \rightarrow Z^{p}(\mathscr{U}, \mathscr{F})$ has dense range.

Here we give an improvement of theorem 1 in a particular case.

COROLlary 2 Let $\pi: X \rightarrow S$ be q-convex and locally q-complete. If $S$ is $r$ complete, then $X$ is cohomologically $(q+r-1)$-complete.

Proof. Let $\mathscr{F} \in \operatorname{Coh}(X)$. One has to check that the cohomology group $H^{q+r-1}(X, \mathscr{F})$ is separated. If $r=1$, this follows from ([11]); for $r \geq 2$ one applies ([10]).

RemarKs. 1) ([10], p. 995]) Let $\pi: X \rightarrow S$ be a $q$-convex morphism. Then the canonical topologies on $H^{i}(X, \mathscr{F}), \mathscr{F} \in \operatorname{Coh}(X)$, are separated for all indices $i \geq q+1$. (No further assumption on $S$ !) It is unknown if this is true for $i=q$.

2) If $\pi: X \rightarrow S$ is locally $q$-convex and $\mathscr{F} \in \operatorname{Coh}(X)$, then the higher direct image sheaf $R^{i} \pi_{*}(\mathscr{F})$ is coherent for all $i \geq q$. However, this and the Leray spectral sequence do not imply our theorem, since $R^{i} \pi_{*}(\mathscr{F})$ for $i<q$ may not be coherent.

The result from theorem 1 is sharp, in fact we show:

EXAMPLe 2. For every positive integers $q, r$, there exists a holomorphic fi- 
bration $\pi$ from $X$ to $S$ with typical fiber $F$ such that $S$ and $F$ are $r$-complete and $q$-complete respectively, and nevertheless, $H^{q+r-1}\left(X, \mathcal{O}_{X}\right)$ does not vanish. (In fact, it has infinite dimension over $\mathrm{C}$.)

Before getting involved with the example, we recall some facts:

$(\bullet)$ Let $\mathscr{F}$ and $\mathscr{G}$ be coherent analytic sheaves on the complex spaces $Y$ and $Z$. We denote by $\mathscr{F} \square \mathscr{G}$ the (coherent analytic) sheaf $p_{Y}^{*} \mathscr{F} \hat{\otimes} p_{Z}^{*} \mathscr{G}$ on $Y \times Z$, where $p_{Y}$ and $p_{Z}$ stands for the canonical projections on $Y$ and $Z$ respectively. E.g. $\mathcal{O}_{Y} \square \mathcal{O}_{Z}=\mathcal{O}_{Y \times Z}$.

$(\bullet)$ The following Künneth formula due to Cassa ([4]) holds. Assume that the cohomology groups $H^{j}(Z, \mathscr{G}), j=0,1, \ldots$, are Hausdorff. Then for every non-negative integer $k$ there exists a topological isomorphism

$$
H^{k}(Y \times Z, \mathscr{F} \square \mathscr{G}) \cong \bigoplus_{i+j=k}\left(\left(H^{i}(Y, \mathscr{F})_{\mathrm{sep}} \hat{\otimes} H^{j}(Z, \mathscr{G})\right) \oplus R_{i j}\right)
$$

where $R_{i j}$ are complex vector space of infinite dimension (with the trivial topology) if $H^{i}(Y, \mathscr{F})$ is not-Hausdorff and $H^{j}(Z, \mathscr{G})$ does not vanish; otherwise $R_{i j}=\{0\}$.

Now, the example goes as follows. Skoda ([14]) produced a locally trivial holomorphic fibration $f: M \rightarrow D$ with fibre $\mathrm{C}^{2}$ and base $D \subset \mathrm{C}$ open, such that $M$ is not Stein. Notice that $H^{1}\left(M, \mathcal{O}_{M}\right)$ is not separated ([7]).

Set $X=M \times\left(\mathrm{C}^{r} \backslash\{0\}\right) \times\left(\mathrm{C}^{q} \backslash\{0\}\right), S=D \times\left(\mathrm{C}^{r} \backslash\{0\}\right)$ and $\pi$ from $X$ to $S$ canonically induced by $f$ and the natural projection on $C^{r} \backslash\{0\}$. It is evident that $\pi$ is a fibre bundle with $q$-complete fibre and $r$-complete base space. Now, the above Künneth formula says that $H^{q+r-1}\left(X, \mathcal{O}_{X}\right)$ is infinitely dimensional; a fortiori $H^{q+r-1}\left(X, \mathcal{O}_{X}\right) \neq 0$.

Here we give some immediate consequences to theorem 1 .

COROLlaRY 3. Every locally q-complete open subset of a r-complete complex space is cohomologically $(q+r)$-complete.

Corollary 4. Let $E \rightarrow S$ be a holomorphic fibre bundle with fiber F. Suppose that $F$ and $S$ are $q$-complete and r-complete respectively. Then $E$ is cohomologically $(q+r)$-complete.

Corollary 5. Let $X$ be a r-complete complex space and $D \subset X$ an open set such that the inclusion map $\iota: D \rightarrow X$ is $q$-convex. Then $D$ is $(q+r-1)$-complete.

Proof, Let $\psi: X \rightarrow \mathrm{R}$ be $r$-convex and exhaustive, and $\varphi: D \rightarrow \mathrm{R}$ the function which gives the $q$-convexity of $\iota$. Then the set $\left\{x \in D ; \varphi(x) \leq c_{\sharp}\right\}$ is closed in $X$; therefore, by standard arguments there exists a smooth rapidly , 
increasing and convex function $\chi: \mathbf{R} \rightarrow \mathbf{R}$ such that the function $\tilde{\varphi}: D \rightarrow \mathbf{R}$ defined by $\tilde{\varphi}=\chi(\psi)+\varphi$ is $(q+r-1)$-convex and exhaustive.

ReMARK. This corollary does not hold for arbitrary $q$-convex mappings. (See the example 1 in $\S 2$.)

The same method used for the proof of theorem 1, together with the subsequent two lemmas

Lemma 6. Let $Y$ be a p-complete complex space of dimension $n$ and $U$ a $p$ Runge domain. Then $H_{i}(Y, U ; G)=0$ for $i \geq n+p$ and every abelian group $G$.

LEMMA 7. Let $Y$ be a p-complete complex space of dimension $n$ which is locally a set theoretic complete intersection and $U$ a p-Runge domain. Then for every abelian group $G, H_{c}^{i}(Y ; G)=0$ for $i \leq n-p$ and the natural map $H_{c}^{n-p+1}(U ; G) \rightarrow H_{c}^{n-p+1}(Y ; G)$ is injective.

from [15] and [16] respectively give us, mutatis mutandis, the following result concerning the vanishing of other cohomology groups on $X$, namely;

TheOREM 2. Let $\pi: X \rightarrow S$ be locally $q$-complete. Let $n=\operatorname{dim}(X)$ and assume that $S$ is $r$-complete. Then, for every abelian group $G$ we have:

(1) $H_{i}(X, G)$ vanishes for $i \geq n+q+r-1$.

(2) $H_{c}^{i}(X, G)$ vanishes for $i \leq n-(q+r)+1$ if $X$ is of pure dimension and locally a set theoretic complete intersection.

(We recall that a complex space $Y$ of pure dimension $n$ is said to be locally a set theoretic complete intersection if each point $y \in Y$ admits a local chart $\iota: V \rightarrow \hat{V} \subset C^{N}$ with $\hat{V}$ open such that $\iota(V) \subset \hat{V}$ is an analytic subset given by precisely $N-n$ equations.)

As an interesting application of our method, we have:

COROllary 6. Let $D$ be a locally q-complete open subset of a r-complete complex space $X$ of dimension $n$. Set $p=q+r-1$. Then $H_{n+p-1}(D ; Z)$ is torsion free and $H_{i}(D ; Z)$ vanishes for $i \geq n+p$. Moreover, if $\partial D$ is real-analytic, then $H_{n+p-1}(D, Z)$ is free.

REMARK. The first part of corollary 6 was proved by Bolondi ([3]) in the case $q=r=1$.

\section{Some remarks on locally 1-convex maps.}

Motivated by what we proved by now, one should ask if there are also similar global finiteness theorems for $X$, when $\pi: X \rightarrow S$ is locally $q$-convex and $S$ enjoys some convexity properties, like $r$-convexity. 
In general, this is not true, e.g. let $\pi: X \rightarrow \mathrm{C}^{n}$ be as in example 1 from $\S 2$ with $A$ an infinite discrete set. Then $\pi$ is 1 -convex, $C^{n}$ is Stein, and $X$ fails to be even cohomologically $(n-1)$-convex. The situation does not improve even if we assume $S$ compact. A simple example is the canonical map $\pi: X=\mathrm{C}^{n} \backslash\{0\} \rightarrow S=\mathrm{P}^{n-1}$, which, of course, is locally 1-convex, and, again $X$ fails to be cohomologically $(n-1)$-convex. (However, without any further assumption on $S$, if $\pi: X \rightarrow S$ is $q$-convex and $\pi(X)$ is relatively compact in $S$, then $X$ is $q$-convex.)

There is one particular case of the situation (\$) considered in the introduction which may be of some interest, namely; Let $\pi: X \rightarrow S$ be locally 1-convex. Then for every $s \in S$ the fiber $X_{s}:=\pi^{-1}(s)$ is a 1-convex space which contains an exceptional compact analytic set $E_{s}$. Put $q_{s}:=\operatorname{dim}\left(E_{s}\right)$ if $X_{s}$ is not Stein; otherwise we take $q_{s}=0$. We assume $q:=q(\pi):=$ $\sup _{s \in S} q_{s}<\infty$.

Recall the relative Stein factorization from ([9]). There exist: a complex space $Y$ together with a proper surjective holomorphic map $\rho: X \rightarrow Y$ such that $\rho_{*}\left(\mathcal{O}_{X}\right)=\mathcal{O}_{Y}$, and Stein morphism $\sigma: Y \rightarrow S$ such that $\sigma \circ \rho=\pi$. Moreover, if $E=\left\{x \in X ; \operatorname{dim}\left(\rho^{-1}(\rho(x))\right)>0\right\}$ denotes the degeneracy set of $\rho$, then the restriction of $\pi$ from $E$ into $S$ is proper. In particular, $\pi(E)$ is an analytic subset of $S$, and the restriction $\sigma$ from $\rho(E)$ onto $\pi(E)$ is a finite map. One checks easily that $q=\sup \left\{\operatorname{dim} \pi^{-1}(\pi(x)) ; x \in E\right\}$.

Proposition 2. If $q>0$, then $X$ is cohomologically $(q+r)$-complete.

Proof. Since $S$ is $r$-complete, $\rho(E)$ is $r$-complete and then $E$ is $(q+r)$ complete ([17]); hence $\rho(E)$ and $E$ have a fundamental systems of $r$-complete and $(q+r)$-complete neighborhoods respectively. By theorem $1, Y$ is cohomologically $(r+1)$-complete; hence from a well-known exact sequence one has $H_{\Phi}^{i}(Y \backslash \rho(E), \mathscr{G})=0$ if $i \geq r+1$ and $\mathscr{G} \in \operatorname{Coh}(Y)$, where " $\Phi$ " means the family of supports made up from all subsets of $Y \backslash \rho(E)$ which are closed in $Y$. Similarly, one gets surjections $H_{\Psi}^{j}(X \backslash E, \mathscr{F}) \rightarrow H^{j}(X, \mathscr{F})$ for $j \geq q+r$ and $\mathscr{F} \in \operatorname{Coh}(X)$, where " $\Psi$ " means the family of supports made up from all suubsets of $X \backslash E$ which are closed in $X$. Since $\pi$ is proper, by Grauert's coherence theorem, $\mathscr{G}:=\rho_{*}(\mathscr{F})$ is coherent. On the other hand, as $\pi$ is closed and $X \backslash E \cong Y \backslash \rho(E)$, we may identify $\Phi$ and $\Psi$. Therefore $H_{*}^{k}(X \backslash E, \mathscr{F}) \cong H_{*}^{k}\left(Y \backslash \rho(E), \rho_{*}(\mathscr{F})\right)$ for every $k$, whence the proposition.

Remark. For $q=0$ we deduce only the cohomological $(r+1)$-completeness of $X$.

Now, we extend a result from [17] to families of 1-convex spaces. 
Proposition 3. If $\pi: X \rightarrow S$ is 1-convex and $S$ is $r$-complete, then $X$ is $(q+r)$-complete.

Proof. (Sketch) We consider $\varphi: X \rightarrow \mathrm{R}$ and $c_{\sharp}$ according to the definition. By replacing $\varphi$ with $\chi(\varphi)$ for a smooth convex function $\chi: \mathbf{R} \rightarrow \mathbf{R}$ such that $\left(-\infty, c_{\sharp}\right]=\{\chi=0\}$ and $\chi$ is strictly increasing on $\left[c_{\sharp}, \infty\right)$, we may assume that $c_{\sharp}=0$ and $\varphi$ is plurisubharmonic on the whole space $X$. For every non-negative real number $c$ we set $X(c):=\{x \in X ; \varphi(x) \leq c\}$.

Let $\psi: S \rightarrow \mathrm{R}$ be $r$-convex and exhaustive. Then for every closed subset $T \subset Y$ such that the restriction of $\sigma$ from $T$ to $S$ is proper, there is a smooth function $f$ on $Y$ which is $r$-convex on $T$. In fact, if $\left\{V_{i}\right\}_{i \in I}$ is a locally finite open covering of $S$ such that $Y_{i}:=\sigma^{-1}\left(V_{i}\right), i \in I$, are Stein, and $f_{i}: Y_{i} \rightarrow \mathrm{R}$ are 1-convex and exhaustive, we put

$$
f:=\phi \circ \sigma+\sum \epsilon_{i}\left(\lambda_{i} \circ \sigma\right) f_{i}
$$

where $\left\{\lambda_{i}\right\}_{i \in I}$ is a partition of unity subordinate to the covering $\left\{V_{i}\right\}_{i \in I}$ and $\epsilon_{i}>0, i \in I$, are sufficiently small constants (which depend on $T$ ).

Now, since $E \subset X\left(c_{\sharp}\right)$ and for $T:=\rho\left(X\left(c_{\sharp}\right)\right)$ with some fixed $c_{*}>c_{\sharp}$, there exists a smooth function $\Phi$ on $X$ which is $(q+r)$-convex on $X\left(c_{*}\right)$, and here $\Phi$ and $\psi \circ \pi+\varphi$ have the same positivity directions ([17]).

Since $\psi \circ \pi+\varphi$ is exhaustive for $X$ and $r$-convex on $X \backslash X\left(c_{\sharp}\right)$, there exist a smooth rapidly increasing convex function $\mu: \mathrm{R} \rightarrow \mathrm{R}$ and $\theta \in C^{\infty}(X, \mathrm{R})$ which equals 1 on $X\left(c_{\sharp}\right)$ and $\sup (\theta) \subset X\left(c_{*}\right)$ such that the function $\mu(\psi \circ \pi+\varphi)+\theta \Phi: X \rightarrow \mathbf{R}$ is $(q+r)$-convex and exhaustive.

ACKNOWLEDGEMENT. This paper was finished when the author held a position at the Institute of Mathematics of the Zürich University.

\section{REFERENCES}

1. A. Andreotti, H. Grauert, Théorèmes de finitude pour la cohomologie des espaces complexes, Bull. Soc. Math. France 90 (1962), 193-259

2. E. Ballico, Finitezza e annullamento di gruppi di coomologia su uno spazio complesso, Boll. Un. Mat. Ital. (6), 1-B (1982), 131-142.

3. G. Bolondi, Homologie des espaces complexes, Singularities, Banach Center Publ. 20, Warsaw 1988, 79-84.

4. A. Cassa, Formule di Künneth per la coomologia a valori in un fascio, Ann. Scuola Norm. Sup. Pisa 27 (1973), 905-931.

5. J.-E. Fornæss, 2-dimensional counterexamples to generalizations of the Levi Problem, Math. Ann. 230 (1997), 169-174.

6. B. Jennane, Groupes de cohomologie d'un fibre holomorphe a base et a fibre de Stein, Invent. Math. 54 (1979), 75-79.

7. B. Jennane, Problème de Levi et morphisme localement de Stein, Math. Ann. 256 (1981), 3742.

8. B. Jennane, Remarques sur les ouverts localement de Stein, Math. Ann. 263 (1983), 371-375. 
9. K. Knorr, M. Schneider, Relativexzeptionelle analytische Mengen, Math. Ann. 193 (1971), 238-254.

10. J.-P. Ramis, Théorèmes de separation et de finitude pour l'homologie et la cohomologie des espaces $(p, q)$ convexes-concaves, Ann. Scuola Norm. Sup. Pisa 27 (1973), 933-997.

11. P. Siegfried, Un théorème de finitude pour les morphismes q-convexes, Comment. Math. Helv. 49 (1974), 417-459.

12. Y.-T. Siu, The 1-convex generalization of Grauert's direct image theorem, Math. Ann. 190 (1971), 203-215.

13. Y.-T. Siu, Dimensions of sheaf cohomology groups under holomorphic deformations, Math. Ann. 192 (1971).

14. H. Skoda, Fibrés holomorphe à base et à fibre de Stein, Invent. Math. 43 (1977), 97-107.

15. V. Vâjâitu, Cohomology with compact supports for q-complete spaces, J. reine angew. Math. 436 (1993), 45-56.

16. V. Vâjâitu, Approximation theorems and homology of q-Runge pairs, J. reine angew. Math. 499 (1994). 179-199.

17. V. Vâjâitu, Some convexity properties of morphisms of complex spaces, Math. Z. 217 (1994), 215-245.

INSTITUTE OF MATHEMATICS OF THE ROMANIAN ACADEMY

P.O. BOX, 1-764.

RO 70700, BUCHAREST

ROMANIA 